\title{
Anesthetic considerations for termination of interstitial pregnancy
}

\begin{abstract}
Background: Interstitial pregnancy, also called cornual pregnancy occurs in $1-4 \%$ of ectopic gestations and predisposes to maternal hemorrhage. Diagnosis can be radiologically difficult.

Case Report: A 30 year old G6P3 female presented with fetal movement after dilation and curettage. MRI was concerning for abdominal pregnancy, fetal age 18 weeks by abdominal girth. Patient was scheduled for exploratory laparotomy under GETA. Pregnancy was found to be cornual and self-contained; patient underwent resection of the ectopic without incident.
\end{abstract}

Conclusion:This case report provides instructive significance for anesthesia management in this rare condition, and development of an anesthetic plan in the face of significant surgical uncertainty.

Keywords: Cornual Pregnancy, Interstitial pregnancy, Anesthesia
Volume 7 Issue 4 - 2017

\section{Lerin E Rutherford, Maya Suresh, Uma \\ Munnur}

Department of Anesthesia, Baylor College of Medicine, USA

Correspondence: Lerin E Rutherford, Baylor College of Medicine, I504 Ben TaubLoop, Houston, TX 77030, USA, Tel 7|3-829-I I I0,Email Iruther@bcm.edu

Received: May 24, 2017 | Published: August II, 2017
Abbreviations: IR, Interventional Radiology; MRI, Magnetic Resonance Imaging; PRBC, Packed Red Blood Cells

\section{Introduction}

Interstitial ectopic pregnancies, also called cornual pregnancies occur in $1-4 \%$ of ectopic gestations. ${ }^{1}$ Risk factors include ipsilateral ectopic pregnancy, salpingectomy and in vitro fertilization. ${ }^{2}$ Usually such pregnancies can advance to around 16 weeks gestation due in part to the greater distensibility of the myometrium surrounding the interstitial segment and distal fallopian tubes. Vascular collaterals develop between the uterine and ovarian arteries to supply the pregnancy, making rupture and hemorrhage a significant risk. ${ }^{3,4}$ Diagnosis can be difficult with imaging; common findings include an empty uterus, separate gestational sac and a thin surrounding myometrial layer. ${ }^{5}$ Corunal pregnancies can be managed with hysterectomy or with medical treatment if the patient is hemodynamically stable.

In contrast, abdominal pregnancies account roughly $1 \%$ of ectopic pregnancies. ${ }^{6,7}$ They often are viable longer than interstitial pregnancies because they are not at risk for rupturing but rarely result in the delivery of a viable fetus. Common complications include severe abdominal pain and massive hemorrhage. Like interstitial pregnancies they can be difficult to diagnose and have high maternal morbidity and mortality.

\section{Case presentation}

A 30 year old G6P3043 African American female with history of previous laparoscopic salpingectomy for ectopic pregnancy was admitted to the obstetrics service after outpatient well-woman exam during which she reported continued sensation of fetal movement after dilation and curettage 8 weeks prior. Transvaginal and transabdominal ultrasound confirmed gestational sac did not communicate with cervical canal. Abdominal MRI was read as consistent with ectopic, possibly abdominal pregnancy, with possible placental invasion into the right internal iliac artery. Fetus was dated $18 w 4 d$ by abdominal circumference, now without fetal heart tones. Patient was scheduled for exploratory laparotomy and removal of abdominal pregnancy (Figure 1).

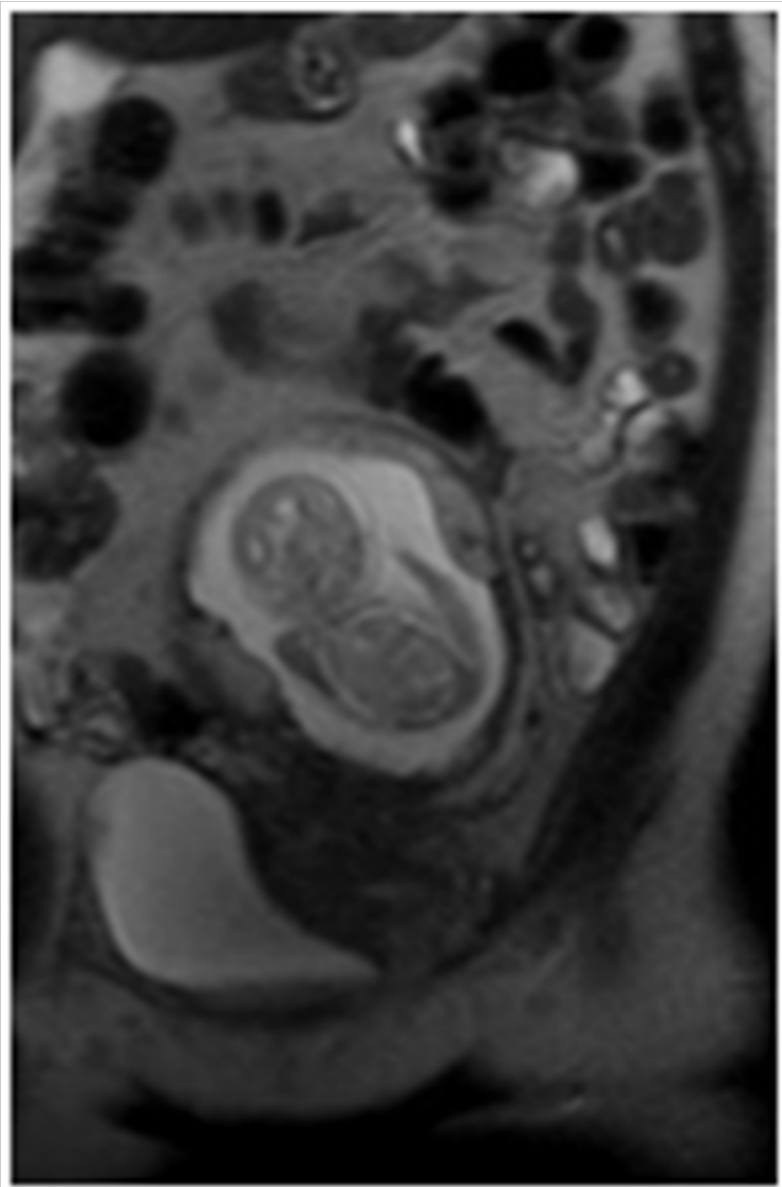

Figure I Abdominal MRI showing fetus superior and exterior to the uterus.

Given potential difficulties of this case, all members of the care team met to discuss anesthetic and surgical plans. Given unclear location of the pregnancy and placenta, hemorrhage was the major concern. If the 
placenta was found to be invading into major vasculature, it would be left in place and treated medically after surgical removal of the fetus. Interventional radiology was consulted and discussed the possibility of preoperative uterine artery embolization and placement of balloon catheters intraoperatively. Due to the risks of the procedure and potential back bleeding of collateral circulation, decision was made to proceed without preoperative intervention. IR remained on standby during the procedure. The intensive care unit was pre-notified that the patient may require advanced care post operatively.

Evaluation by anesthesia revealed Mallampati III airway with good distances, full range of motion and good oral aperture. Patient was 5'4" with a body mass index of 36 . Preoperative workup revealed normal hemoglobin and hematocrit, normal coagulation profile and unremarkable chemistries. Urine drug screen was positive for cannabinoids. Review of systems revealed tobacco use and uncontrolled gastroesophageal reflux disease. Patient was classified ASA 3 (Figure 2).

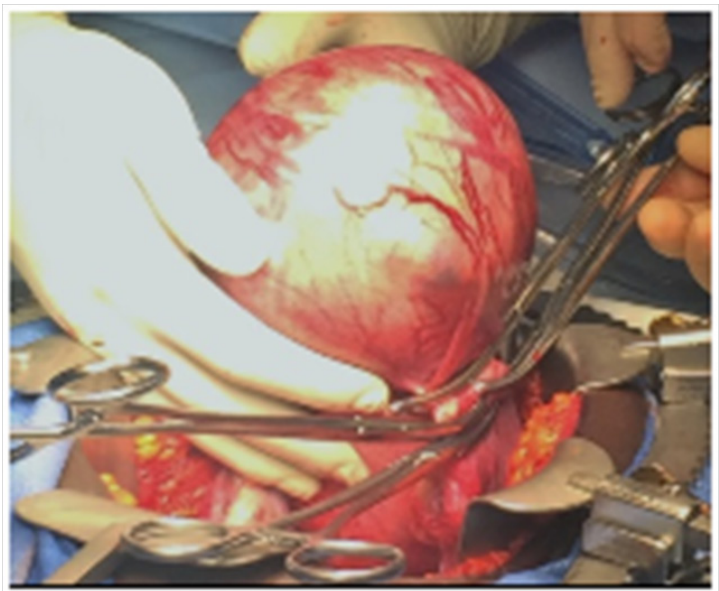

Figure 2 Cornual Pregnancy externalized from the abdomen, uterus intact below.

Prior to induction, patient was typed and crossed for 4 units. Blood bank was notified of potential massive transfusion protocol. Patient had one 16 gauge IV placed preoperatively. Given increased aspiration risk in pregnant patients, she received bicitra and famotidine prophylaxis. Patient underwent rapid sequence intubation with cricoid pressure via direct laryngoscopy. She was maintained on general endotracheal anesthesia. Radial artery catheter was placed for invasive blood pressure monitoring. Patient received a 12 French triple lumen catheter in the right internal jugular. Four units typed and crossed $\mathrm{pRBCs}$ were present in the room with warmers and blood sets. Patient received prophylactic antibiotics prior to incision.

Intraoperatively patient was stable requiring minimal pressor support. Upon opening, pregnancy was found to be cornual and self-contained. Surgery proceeded without incident and the ectopic pregnancy was resected. Pregnancy specimen measured over 13 $\mathrm{cm}$ in diameter. Estimated blood loss was $200 \mathrm{~mL}$. Patient received $1.9 \mathrm{~L}$ crystalloid and $250 \mathrm{~mL}$ albumin. She received ondansetron and metoclopramide as prophylaxis for post-operative nausea and vomiting. Patient had an uneventful post-operative course and was discharged home on post-operative day 3 (Figure 3).

\section{Discussion}

Given the rarity of such cases, description of anesthetic management in the literature is limited. This case was uneventful surgically as the configuration of the pregnancy enabled easy resection. Given the ambiguity of the preoperative imaging, multiple surgical approaches may have been necessary; the anesthesia plan had to account for all of the possibilities. Possible challenges that might have arisen included invasion of the placenta into surrounding structures requiring assistance from general surgery or embolization of the uterine artery by IR requiring transport of the patient to the radiology suite or conversion to a staged procedure. Development of a safety-minded anesthetic plan required input and coordination from multiple specialties. Appropriate communication played an enormous role in the success of this operation.

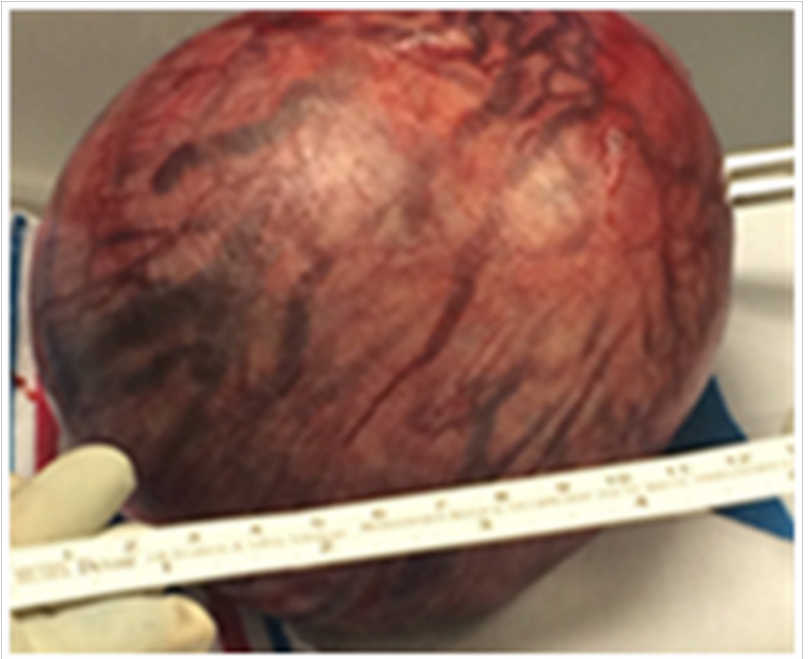

Figure 3 Cornual pregnancy resected with ruler for scale, over $13 \mathrm{~cm}$ in diameter.

Both interstitial and abdominal pregnancies are known to cause massive hemorrhage, perhaps the most feared complication in this case. The anesthetic management plan included full preparation for transfusion. The team alerted the blood bank of the potential for massive transfusion protocol activation and delegated this task to a specific OR staff member. Warmers, blood sets, and four units pRBCs were already present in the room. Good IV access and invasive blood pressure monitoring were essential.

Surgery in pregnant patients brings up an additional set of considerations. Increased airway edema and soft tissue mass can make airway management difficult in pregnant patients. Although intubation was achieved with direct larygoscopy, a CMAC video larygoscope was present in the room. Pregnant patients also experience increased aspiration risk due to delayed gastric emptying, relaxation of the gastroesophageal sphincter, and increased intragastric pressures due to the gravid uterus. The patient was pretreated with famotidine and cricoid pressure was applied throughout rapid sequence intubation. In this case since the fetus was non-viable the anesthetic plan did not have to account for transfer for anesthetic agents across the placenta.

Complex and high risk cases such as these benefit from a team based approach and meticulous preparation for anticipated issues. Strategies such as delegating tasks, having all anticipated equipment present in the room and having assistance (surgical, nursing and anesthesia) on standby are invaluable.

\section{Acknowledgements}

None. 


\section{Conflicts of Interest}

None.

\section{References}

1. Tang A, Baartz D, Khoo SK. A medical management of interstitial ectopic pregnancy: a 5-year clinical study. Aust N Z JObstet Gynaecol. 2006;46(2):107-111.

2. Tulandi T, Al Jaroudi D. Interstitial pregnancy: results generated from the Society of Reproductive Surgeons Registry. Obstet Gynecol. 2004;103(1):47-50.

3. Kun W, Tung W. On the lookout for a rarity: interstitial/ cornual pregnancy. Eur J Emer Med. 2001;8(2):147-150.
4. Thakur Y, Coker A, Morris J, et al. Laparoscopic and ultrasound-guided transcervicalevacuation of cornual ectopic pregnancy: an alternative approach. Obstet Gynaecol. 2004;24(7):809-810.

5. Timor-Tritsch IE, Monteagudo A, Matera C, et al. Sonographic evolution of cornual pregnancies treated without surgery. Obstet Gynecol. 1992;79(6):1044-1046.

6. Nwobodo EI. Abdominal pregnancy. A case report. Ann Afr Med. 2004;3(4):195-196.

7. Badria L, Amarin Z, Jaradat A, et al. Full-term viable abdominal pregnancy. A case report and review. Arch Gynaecol Obstet. $2003 ; 268(4): 340-342$. 